\title{
Effect of palladium doping on the stability and fragmentation patterns of cationic gold clusters
}

\author{
P. Ferrari, ${ }^{1}$ H. A. Hussein,${ }^{2,3}$ C. J. Heard, ${ }^{4}$ J. Vanbuel, ${ }^{1}$ R. L. Johnston, ${ }^{2}$ P. Lievens, ${ }^{1}$ and E. Janssens ${ }^{1}, *$ \\ ${ }^{1}$ Laboratory of Solid State Physics and Magnetism, KU Leuven, 3001 Leuven, Belgium \\ ${ }^{2}$ School of Chemistry, University of Birmingham, Edgbaston, Birmingham B15 2TT, United Kingdom \\ ${ }^{3}$ Department of Chemistry, College of Science, University of Kufa, Najaf, Iraq \\ ${ }^{4}$ Department of Physical and Macromolecular Chemistry, Charles University, 12843 Praha2, Czech Republic
}

(Received 12 December 2017; published 21 May 2018)

\begin{abstract}
We analyze in detail how the interplay between electronic structure and cluster geometry determines the stability and the fragmentation channels of single Pd-doped cationic Au clusters, $\mathrm{PdAu}_{N-1}+(N=2-20)$. For this purpose, a combination of photofragmentation experiments and density functional theory calculations was employed. A remarkable agreement between the experiment and the calculations is obtained. Pd doping is found to modify the structure of the Au clusters, in particular altering the two-dimensional to three-dimensional transition size, with direct consequences on the stability of the clusters. Analysis of the electronic density of states of the clusters shows that depending on cluster size, Pd delocalizes one $4 d$ electron, giving an enhanced stability to $\mathrm{PdAu}_{6}{ }^{+}$, or remains with all $4 d^{10}$ electrons localized, closing an electronic shell in $\mathrm{PdAu}_{9}{ }^{+}$. Furthermore, it is observed that for most clusters, Au evaporation is the lowest-energy decay channel, although for some sizes Pd evaporation competes. In particular, $\mathrm{PdAu}_{7}{ }^{+}$and $\mathrm{PdAu}_{9}{ }^{+}$decay by Pd evaporation due to the high stability of the $\mathrm{Au}_{7}{ }^{+}$and $\mathrm{Au}_{9}{ }^{+}$fragmentation products.

DOI: 10.1103/PhysRevA.97.052508
\end{abstract}

\section{INTRODUCTION}

The search for stable cluster species has been an important subject in the study of small metallic clusters ever since the discovery that the stability of a cluster strongly depends on the number of constituent atoms. In 1984, Knight et al. [1] reported the surprising result that Na clusters composed of 8, 20, 40, and 58 atoms are significantly more stable than other sizes, describing them as "magic number" clusters. The observed stability pattern could be explained by phenomenological electronic shell models [2]. Electronic shells result from the confinement of delocalized valence electrons (one for each $\mathrm{Na}$ atom) within the small volume of the cluster. For a perfectly spherical "particle in a box," $1 S, 1 P, 1 D, 2 S, 1 F, 2 P, \ldots$ shells are formed, explaining the enhanced stability of the magic clusters, in the same way that noble gases are stable because of their closed electronic shells. The term "superatom" is sometimes adopted for this reason [3]. The geometry of most clusters, however, is quite different from a perfect sphere. A consequence of a lower structural symmetry is that electronic shell degeneracies are lifted, resulting in a subshell structure [4]. In quasi-two-dimensional (quasi-2D) structures, new magic numbers emerge by energetic destabilization of out-of-plane orbitals [5]. For example, six (closure of $1 S$ and $1 P_{x, y}$ shells) is a magic number for electrons that are confined in a two-dimensional triangular potential well [6]. The identification of stable clusters and the understanding of the interplay between localization and delocalization of valence electrons is of high importance for the development of novel applications using size-selected clusters.

\footnotetext{
*ewald.janssens@kuleuven.be
}

Small gold clusters are ideal model systems to investigate the interplay between electronic magic numbers and cluster geometry. The heavy gold atom is subjected to strong relativistic effects, which reduce the $5 d-6 s$ energy separation, with direct consequences for the properties of gold clusters [7]. $5 d$ electrons are partially involved in the bonding between the atoms and thus gold clusters adopt structures very different from clusters of other elements [8]. For example, ion mobility experiments have shown that negatively charged gold clusters are planar up to $N=11$ [9], whereas recent calculations predicted that neutral species can be planar up to the surprisingly large number of 13 atoms [10]. For positively charged clusters, the $2 \mathrm{D}$ to $3 \mathrm{D}$ transition is known to take place at $N=9$, with $\mathrm{Au}_{8}{ }^{+}$the largest planar cluster [11].

These special features have consequences for the sizedependent stability pattern of gold clusters; electronic shell degeneracies associated with angular momentum are lost (while maintaining spin pairing) because of their rather asymmetric structures, and thus subshells open and close every time an atom (and, therefore, an electron) is added to the system. Hence, stability patterns of gold clusters depend strongly on size, possessing pronounced odd-even variations [12-14]. Interestingly, even though the hybridization between the $d$ and $s$ states in Au clusters is important, due to relativistic effects, simple electron counting rules for the valence electrons can explain their stability patterns quite well [15]. Another surprising feature of gold clusters is their reactivity. Even though bulk gold is one of the most noble elements of the periodic table, at the nano- and subnanoscale, Au particles become reactive and can act as catalysts for specific reactions, such as CO oxidation [16], methane activation [17], and hydrogen dissociation [18]. The stability of the catalyst is clearly of high importance. 
Doping can significantly influence the stability of a cluster; first, by altering the system's atomic structure, thus modifying the shape of the "box" confining the delocalized electrons, and second, by changing the number of valence electrons available for delocalization, which may lead to new shell closings $[12,19,20]$. From a fundamental point of view, Pd doping is a very interesting case for study because of its ground-state electronic configuration, with a closed $4 d$ shell and no valence $s$ electrons $\left([\mathrm{Kr}] 4 d^{10}\right)$. Photoelectron spectroscopy measurements on small Pd-doped anionic gold clusters, $\mathrm{PdAu}_{N}{ }^{-}(N=1-4)$, suggested that the Pd-dopant atom is excited to a $[\mathrm{Kr}] 4 d^{9} 5 s^{1}$ state, after which the Pd valence $s$ electron is delocalized and participates in the metallic bonding [21]. In addition, density functional theory (DFT) calculations on several cluster sizes in different charge states have shown that Pd doping can strongly influence both the atomic and the electronic structures of gold clusters [22-24]. Here we study the effect of Pd doping on the size-dependent stability of gold clusters. Pd doping is also interesting from a more applied point of view. Various studies have shown the influence of $\mathrm{Pd}$ in diverse properties of Au clusters, such as a quenching effect of the optical absorption in the visible range [25,26], an increase in the adsorption energies of molecules such as $\mathrm{CO}$ and $\mathrm{O}_{2}$ [27], or an enhancement of the catalytic properties toward a variety of reactions [28-30]. Moreover, significant efforts have been devoted to the production and study of the properties of monolayer-protected Pd-doped Au clusters [31-35]. In all these clusters, the modification of the electronic structure upon doping plays a major role.

In this work, we combine mass spectrometric experiments with DFT calculations to investigate the effect of Pd doping on the stability patterns and the electronic structures of cationic $\mathrm{Au}_{N}{ }^{+}(N=2-20)$ clusters. The stability patterns of the pure and doped clusters as well as the preferred fragmentation channels are studied by photofragmentation. An extensive search for minimum-energy structures was conducted using DFT. The computational results are compared with the experimental findings, allowing better insight into the interplay between geometry and electronic structure of the clusters and the consequences thereof for their relative stability.

\section{METHODS}

\section{A. Mass spectrometric experiments}

Pure and Pd-doped $\mathrm{Au}_{N}{ }^{+}$clusters are produced by laser ablation and inert gas condensation. Their size distribution is analyzed by time-of-flight mass spectrometry, in a setup described in detail in Ref. [36]. A molecular beam containing both charged and neutral clusters is produced, but charged species are electrostatically removed from the beam. Only neutral species enter the mass spectrometer, where they are excited by a tightly focused excimer $F_{2}$ laser $(\lambda=157 \mathrm{~nm})$, which triggers ionization and fragmentation. While the abundance distribution of clusters as produced in the source is strongly influenced by production conditions, such as ablating laser energy and carrier gas pressure, this is different for the spectra recorded after photofragmentation. The abundance of a specific cluster size relative to neighboring sizes in a photofragmentation experiment is sensitive to the relative dissociation energy of that cluster, and thus reflects its relative stability [37,38].

\section{B. Theoretical calculations}

An extensive search for minimum-energy structures (at the DFT level of theory) was performed for monometallic $\mathrm{Au}_{N}{ }^{+}$and $\mathrm{Pd}$-doped $\mathrm{PdAu}_{N-1}{ }^{+}$clusters, in the extended $N=2-20$ size range. This search was carried out using the Birmingham Parallel Genetic Algorithm (BPGA) [39,40], which employs a pool methodology to evaluate structures in parallel. In each run, multiple BPGA instances are implemented and, in each instance, a set of processes is run in parallel and independently [41,42]. First, numerous random geometries are generated to form a population [43]. Then, the generated structures of a given population are relaxed, followed by crossover and mutation operations being performed on individuals in the population. The newly generated structures are locally energy minimized at the DFT level and then the highest-energy isomers are replaced by any lower-energy isomers among the set of offspring and mutants. All the local energy minimizations mentioned above were conducted with Gamma-point DFT calculations employing the Vienna ab initio Simulation Package (VASP) [44]. Projected-augmented wave (PAW) pseudopotentials were used, with the generalized gradient approximation (GGA) Perdew-Burke-Ernzerhof (PBE) exchange-correlation functional [45,46]. A plane-wave basis set was implemented including spin polarization. The plane-wave cut-off energy was truncated at $400 \mathrm{eV}$. MethfesselPaxton smearing, with a sigma value of $0.01 \mathrm{eV}$, was implemented to improve convergence [47]. The thresholds for the electronic energy and forces were set to $10^{-6} \mathrm{eV}$ and $10^{-5} \mathrm{eV} / \AA$, respectively. Spin states were optimized within VASP independently for each generated global minimum from BPGA. All clusters were found to exhibit the lowest possible spin state in the optimal electronic configurations. Finally, the energies of the lowest-energy VASP structures were recalculated using spin-unrestricted density functional calculations within the NWCHEM software package [48], employing the long-range-corrected LC- $\omega$ PBEh exchangecorrelation functional and the extensive def2-TZVPP basis set within the corresponding effective core potential (def2-ECP) of Weigend and Ahlrichs [49-51].

\section{RESULTS AND DISCUSSION}

\section{A. Photofragmentation results}

Mass spectra of pure $\mathrm{Au}_{N}{ }^{+}$and doped $\mathrm{PdAu}_{N}{ }_{-1}^{+}$clusters after photofragmentation are shown in Fig. 1. Stability patterns are clearly visible. For monometallic Au clusters, strong oddeven alterations in intensity are present, with higher intensities for clusters with an odd number of atoms (even number of $6 \mathrm{~s}$ electrons). This pattern has been observed previously and is well understood in terms of the electronic shell model $[2,12]$. Two distinct maxima are seen for the photofragments $\mathrm{Au}_{7}{ }^{+}$and $\mathrm{Au}_{9}{ }^{+}$, which possess stable closed electronic shells with six and eight delocalized electrons, respectively (see discussion later) $[4,6]$. For positively charged gold clusters, the 2D to 3D transition takes place at size $N=9$, with $\mathrm{Au}_{8}{ }^{+}$being the largest planar cluster [11]. Additional, low-intensity signals on 


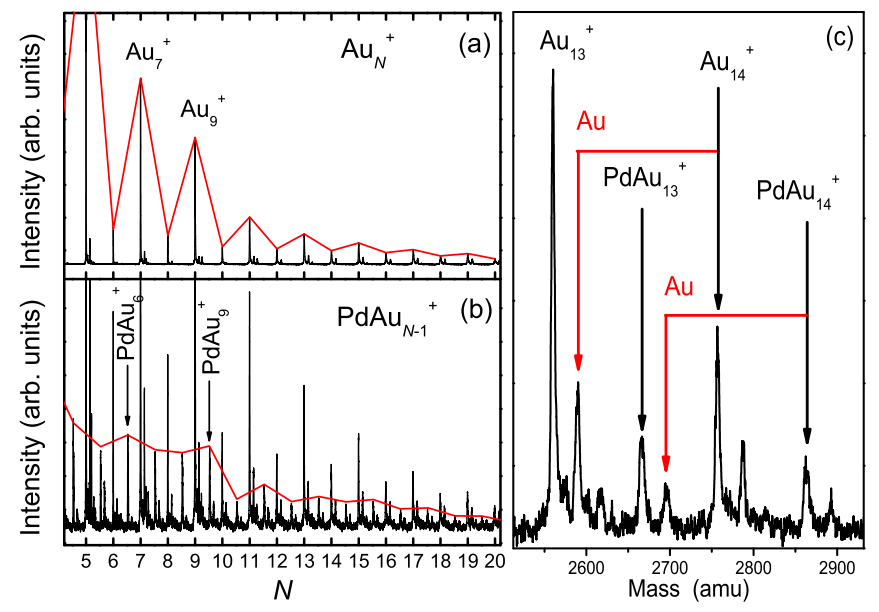

FIG. 1. Mass abundance spectra of clusters after photofragmentation with a tightly focused $F_{2}$ excimer laser $(\lambda=157 \mathrm{~nm})$. (a) $\mathrm{Au}_{N}{ }^{+}$clusters. (b) $\mathrm{PdAu}_{N-1}{ }^{+}$clusters. Peaks are connected by a red line as a guide for the eye to visualize the size-to-size variations in intensity. (c) Zoom-in on (b) highlighting the pathways for metastable fragmentation of $\mathrm{Au}_{14}{ }^{+}$and $\mathrm{PdAu}_{14}{ }^{+}$.

the right-hand side of each $\mathrm{Au}_{N}{ }^{+}$cluster in the mass spectrum correspond to metastable fragments, as discussed later.

The mass spectrum of photofragmented $\mathrm{PdAu}_{N-1}{ }^{+}$clusters [see Fig. 1(b)] demonstrates that the stability pattern is significantly altered by $\mathrm{Pd}$ doping. The most pronounced intensity drop is now found after size $N=10$, instead of after $N=9$ for the monometallic $\mathrm{Au}_{N}{ }^{+}$clusters. This suggests that $\mathrm{PdAu}_{9}{ }^{+}$has a relatively enhanced stability. Odd-even oscillations are seen in the abundances, but are less pronounced than for the monometallic gold clusters. For $N>8$, the higher intensities are found for $\mathrm{PdAu}_{N-1}{ }^{+}$clusters composed of an even number of atoms, while for $\mathrm{Au}_{N}{ }^{+}$odd- $N$ sizes are most intense. Replacing an odd-electron Au atom by an evenelectron $\mathrm{Pd}$ atom means that even- $N$ cationic clusters now have an even number of valence electrons. Interestingly, this odd-even pattern is broken below $N=8$, with a local intensity maximum at $\mathrm{PdAu}_{6}{ }^{+}$, a cluster containing an odd number of atoms (and electrons).

Following multiple photon absorption, the internal energy of a cluster increases significantly, triggering fragmentation as a cooling (relaxation) mechanism [52]. Initially, fragmentation proceeds at a very high rate and thus the first fragmentation step takes place on a much faster time scale than the time scale of the time-of-flight mass spectrometry experiment (which is of the order of several microseconds). The photoexcited clusters are thus accelerated with the mass of those "instantaneous" fragmentation products. If no additional fragmentation steps occur before detection, the fragment is denoted as "prompt." These instantaneous or prompt fragments correspond to the species with the higher intensities in Fig. 1 and their size-tosize intensity variations reveal the electronic shell structure, as discussed above. Besides these prompt fragments, some clusters present in the molecular beam after photoexcitation are metastable. Such a cluster is the product of subsequent fragmentation steps, taking place on much longer time scales than prompt fragmentation because the internal energy of the cluster is now much lower [53]. A metastable fragment is recognizable in the mass spectrum as an additional peak next to the prompt clusters, as shown in Fig. 1(c). The apparently different mass of a metastable cluster is just a consequence of the fact that fragmentation taking place in free flight slightly changes the time of flight of the ion in the reflectron of the mass spectrometer (see Supplemental Material [54] for details).

Even though cluster stabilities can be investigated by the intensity of prompt fragments in a mass spectrum, information about the decay channel producing the fragments cannot be obtained by this approach. This is because the time of flight of a prompt fragment only depends on its mass after fragmentation. However, this is different for a metastable cluster, with a time of flight that depends on both the flight times of mother and daughter ions in the fragmentation process [53]. An example can be found in Fig. 1(c), which shows a zoom-in in Fig. 1(b). For instance, the decay channel $\mathrm{Au}_{14}{ }^{+} \rightarrow \mathrm{Au}_{13}{ }^{+}+\mathrm{Au}$ leads to a metastable peak next to the prompt $\mathrm{Au}_{13}{ }^{+}$cluster. Similarly, the fragmentation channel $\mathrm{PdAu}_{14}{ }^{+} \rightarrow \mathrm{PdAu}_{13}{ }^{+}+\mathrm{Au}$ is identified by the presence of a metastable fragment visible after the prompt $\mathrm{PdAu}_{13}{ }^{+}$cluster. A metastable fragment produced by the channel $\mathrm{PdAu}_{14}{ }^{+} \rightarrow \mathrm{Au}_{14}{ }^{+}+\mathrm{Pd}$ would show in between the prompt $\mathrm{Au}_{14}{ }^{+}$and the metastable $\mathrm{Au}_{15}{ }^{+}$peaks, which is not observed. Thus, depending on the flight time of the metastable clusters, the decay channels can be identified.

\section{B. Theoretical results}

The lowest-energy structures obtained for $\mathrm{Au}_{N}{ }^{+}$and $\mathrm{PdAu}_{N-1}{ }^{+}(N=2-20)$ are shown in Fig. 2 and Cartesian coordinates are provided as supporting information. The cationic pure $\mathrm{Au}_{N}{ }^{+}$geometries can be identified as planar fragments of hexagonal layers for $N$ up to 8 atoms, 3D relaxed fcc bulk fragments for $N=9-13$, and 3D hollow cage structures for $N=14-20$. The obtained structures agree well with those found by combined ion mobility experiments and DFT calculations (in the $N<14$ size range) [11], except for $\mathrm{Au}_{8}{ }^{+}$. For $\mathrm{Au}_{8}{ }^{+}$, a planar structure is predicted as the global minimum in our calculations, whereas the experiment assigned a 3D isomer as the species present in the molecular beam. Similar findings have been found elsewhere [55]. This 3D isomer has a relative energy of $0.023 \mathrm{eV}$ in our calculations. Although this energy lies within the accuracy of DFT, possibly explaining the discrepancy, another possibility is that the ground-state structure is not the cluster produced in the experiment. Additional theoretical studies on the structures of cationic $\mathrm{Au}$ clusters can be found in the literature, although not for the entire size range presented here. Previously reported global minima agree in general with our results [56-59]. Compared with our recent global optimizations of neutral palladium-gold clusters $[10,60]$, there is a significant effect of charge on the structural patterns. Losing one electron in pure neutral $\mathrm{Au}_{N}$ clusters is responsible for a shift of the 2D-3D crossover from $N=14$ down to $N=9[10,60,61]$.

Pd doping significantly alters the structures of the cationic $\mathrm{Au}$ clusters. The most remarkable structural change is the large decrease in the $2 \mathrm{D}$ to $3 \mathrm{D}$ transition size, which for the doped clusters is at the smallest possible size: $N=4$. $\mathrm{PdAu}_{3}{ }^{+}$has a tetrahedral geometry and all larger Pd-doped clusters are three dimensional, in clear contrast with $\mathrm{Au}_{N}{ }^{+}$. 

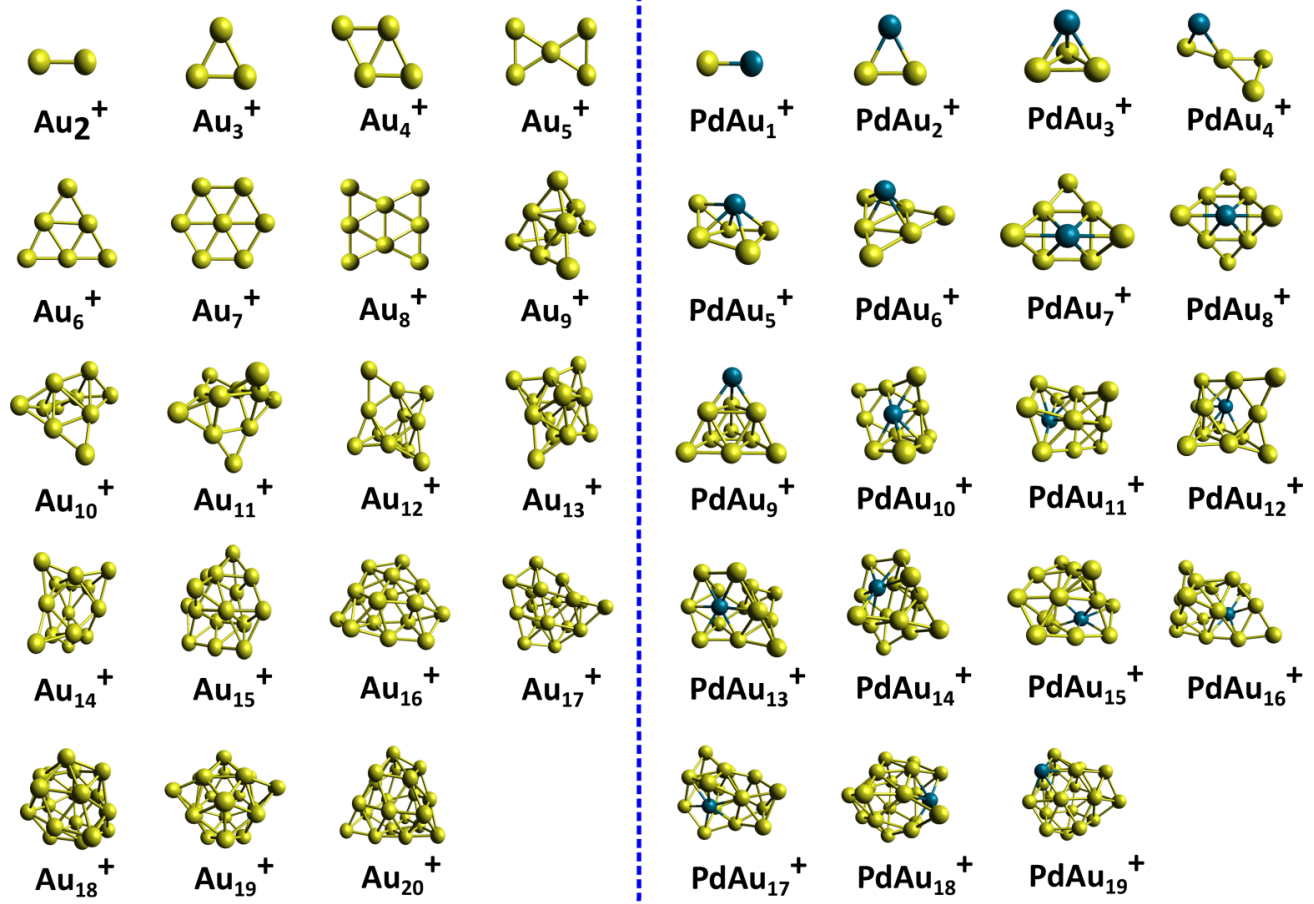

FIG. 2. Minimum-energy structures of $\mathrm{Au}_{N}{ }^{+}$and $\mathrm{PdAu}_{N-1}{ }^{+}(N=2-20)$ clusters. Au atoms are represented in yellow and $\mathrm{Pd}$ in blue.

This dopant-induced dimensional transition is consistent with previous studies [23,24]. Our predicted isomers satisfactorily concur with those presented by previous theoretical studies on Pd-doped Au clusters up to six atoms [25,62,63]; however, two differences are observed in the case of $\mathrm{PdAu}_{4}{ }^{+}$and $\mathrm{PdAu}_{5}{ }^{+}$clusters. Our $\mathrm{PdAu}_{4}{ }^{+}$structure is found to show a 3D "twisted bow tie" as previously reported in Ref. [25], which does not agree with the $M$-like planar structure proposed in Ref. [63] or with the trigonal bipyramid structure suggested in Ref. [62]. The global minimum of $\mathrm{PdAu}_{5}{ }^{+}$is found to be a capped square-based pyramid, while an edge-bridged trigonal bipyramid was previously proposed as the groundstate structure for this cluster [62]. For larger $\mathrm{PdAu}_{m}{ }^{+}(m>6)$ clusters, we are unaware of theoretical or experimental studies to identify their detailed structures. For all sizes, the Pd atom occupies a high-coordination site in or close to the center of the cluster, as previously observed for the neutral species $[10,60]$.

\section{Stability patterns}

Under the assumption that an evaporative decay chain proceeds through successive emission of monomers, the quantity $\ln \left(I_{N} / I_{N+1}\right)$, with $I_{N}$ corresponding to the intensity of cluster size $N$, provides information about relative stabilities $[37,38]$. This is different from the case in which cluster distributions are produced by a thermodynamic (quasi)equilibrium between growth and dissociation [4]. Values of $\ln \left(I_{N} / I_{N+1}\right)$, as a function of cluster size, are plotted in Fig. 3 for the monometallic $\mathrm{Au}_{N}{ }^{+}$[Fig. 3(a)] and doped $\mathrm{PdAu}_{N-1}{ }^{+}$[Fig. 3(c)] clusters. From this figure, the previously mentioned size-to-size patterns can be discussed quantitatively; strong odd-even oscillations are found for $\mathrm{Au}_{N}{ }^{+}$, with pronounced maxima for $\mathrm{Au}_{7}{ }^{+}$and $\mathrm{Au}_{9}{ }^{+}$, and local maxima for clusters composed of an odd number of atoms (even number of valence electrons). For the doped species, a maximum is found for $\mathrm{PdAu}_{9}{ }^{+}$, after which odd-even variations are present, with local maxima for clusters composed of an even number of atoms. For clusters smaller than $\mathrm{PdAu}_{8}{ }^{+}$, the pattern changes and a local maximum appears at $\mathrm{PdAu}_{6}{ }^{+}$. The reason for the deviation from the odd-even alternation in the $N=6-8$ region for the Pd-doped clusters (both from theory and experiment) will be discussed later.

The observed stability pattern of the $\mathrm{PdAu}_{N-1}{ }^{+}$clusters (with $N>8$ ) can be described in terms of the electronic shell model. Assuming that all $d$ electrons of the Pd-dopant atom (electronic configuration $[\mathrm{Kr}] 4 d^{10}$ ) remain localized and that each $\mathrm{Au}$ atom delocalizes its $6 s$ electron (one of which is lost in the cationic charge state), clusters with an even number of atoms have an even number of delocalized electrons and correspond to closed shells of itinerant electrons. In this interpretation, the stability maximum for $\mathrm{PdAu}_{9}{ }^{+}$corresponds to a $3 \mathrm{D}$ magic number of electrons (eight), provided that $\mathrm{PdAu}_{9}{ }^{+}$indeed has a 3D geometry, as the DFT calculations suggest (see Fig. 2). The stability pattern observed below $N=8$ is less easy to interpret and requires a detailed analysis of the electronic structure of the clusters.

Information on the stability of a cluster relative to the neighboring sizes can be obtained from theory by calculating the second difference in energy $\left(\Delta_{2} E\right)$. For a cluster of size $N$, this quantity is defined as

$$
\Delta_{2} E=E\left(A_{N+1}^{+}\right)+E\left(A_{N-1}^{+}\right)-2 E\left(A_{N}^{+}\right),
$$

where $A_{N}{ }^{+}$is the cluster composed of $N$ atoms and $E\left(A_{N}{ }^{+}\right)$is its total electronic energy. With this definition of $\Delta_{2} E$, a cluster which is more stable than its closest neighbors has a positive $\Delta_{2} E$ value. This quantity, as a function of cluster size, is plotted in Fig. 3 for $\mathrm{Au}_{N}{ }^{+}$[Fig. 3(b)] and PdAu ${ }_{-1}^{+}$[Fig. 3(d)]. 


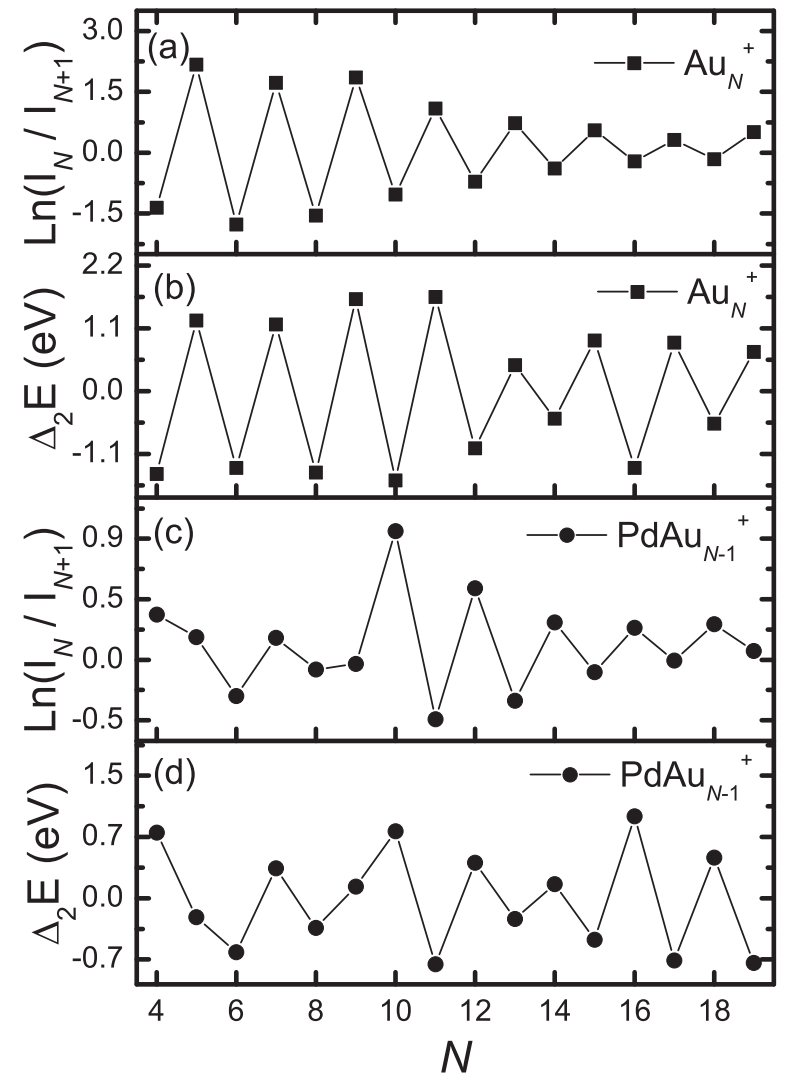

FIG. 3. Relative stability patterns of $\mathrm{Au}_{N}{ }^{+}$and $\mathrm{PdAu}_{N-1}{ }^{+}$ clusters. Experimentally, stabilities are quantified by the quantity $\ln \left(I_{N} / I_{N+1}\right)$, presented in (a) for $\mathrm{Au}_{N}{ }^{+}$and in (c) for $\mathrm{PdAu}_{N-1}{ }^{+}$. Theoretically, relative stabilities are characterized by second differences in energy $\left(\Delta_{2} E\right)$, obtained from the density functional theory calculations. These are shown in (b) for $\mathrm{Au}_{N}{ }^{+}$and in (d) for $\operatorname{PdAu}_{N-1}{ }^{+}$.

The abundance variations found in the photofragmentation experiment are reproduced very well by $\Delta_{2} E$. Important features are as follows: (i) odd-even oscillations are present for all the $\mathrm{Au}_{N}{ }^{+}$clusters, with higher stability for clusters composed of an odd number of atoms. (ii) Maxima are seen for $\mathrm{Au}_{9}{ }^{+}$and $\mathrm{Au}_{7}{ }^{+}$. (iii) Upon Pd doping, odd-even oscillations are present for $N>8$, with maxima for even $N$. (iv) The most pronounced maximum for the Pd-doped clusters corresponds to $\mathrm{PdAu}_{9}{ }^{+}$. (v) The odd-even pattern is broken for the Pd-doped clusters when $N<8$, with a local maximum at $\mathrm{PdAu}_{6}{ }^{+}$. Taking into account the assumptions underlying Eq. (1), the agreement between theory and experiment is exceptional.

The decay channels for the fragmentation of $\mathrm{Au}_{N}{ }^{+}$and $\mathrm{PdAu}_{N-1}{ }^{+}$clusters, investigated through the measurement of metastable fragments, are compared in Fig. 4. Metastable fragmentation, as a function of cluster size, is characterized by the ratio of the intensity of metastable to prompt clusters of the same size $\left(M_{N}\right)$. The decay channels of monometallic $\mathrm{Au}_{N}{ }^{+}$ clusters are known [12]; depending on cluster size, dimer loss competes with monomer evaporation. Figure 4(a) shows that $\mathrm{Au}_{N}{ }^{+}$clusters composed of an even number of atoms decay only by monomer evaporation, whereas those composed of an odd number of atoms decay by both channels. This odd-even pattern in the decay channels of the clusters can be explained

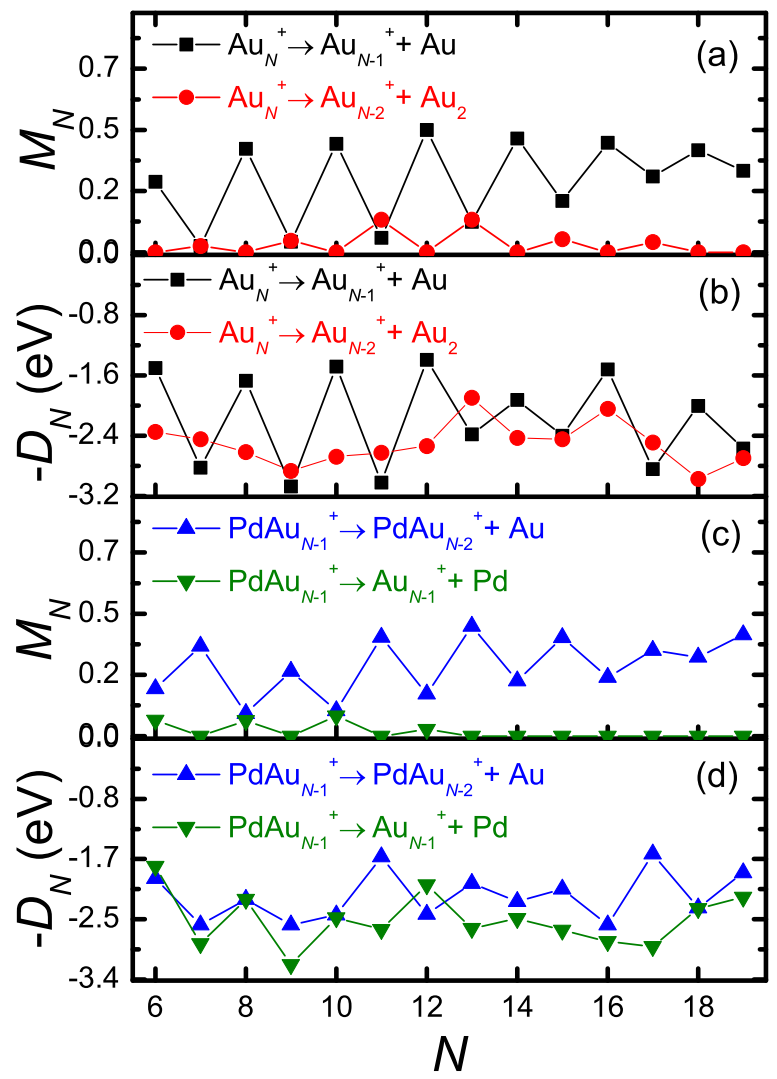

FIG. 4. Metastable fractions $\left(M_{N}\right)$ obtained by the photofragmentation experiments. (a) Metastable fraction corresponding to monomer or dimer evaporation for the monometallic $\mathrm{Au}_{N}{ }^{+}$clusters. (c) Metastable fraction corresponding to $\mathrm{Au}$ or Pd evaporation for $\mathrm{PdAu}_{N-1}{ }^{+}$clusters. (b) Dissociation energies, as calculated by DFT, for monometallic $\mathrm{Au}_{N}{ }^{+}$clusters corresponding to the removal of a neutral monomer or dimer. (d) Dissociation energies, as calculated by DFT, for Pd-doped $\mathrm{PdAu}_{N-1}{ }^{+}$clusters corresponding to the removal of a neutral $\mathrm{Au}$ or $\mathrm{Pd}$ atom.

by their stability. When $N$ is even, monomer evaporation is the preferred channel so that the fragmentation product is an odd-atom cationic cluster with a closed electronic shell (even number of electrons). Conversely, if $N$ is odd, dimer evaporation is the channel which generates a closed-shell fragment cluster. For the Pd-doped clusters, no dimer evaporation is observed (above the noise level of the measurement), while Pd monomer evaporation is a competitive decay channel with $\mathrm{Au}$ evaporation for some sizes. Figure 4(c) shows the metastable fractions for the doped $\mathrm{PdAu}_{N-1}{ }^{+}$clusters. Pd evaporation is observed for $\mathrm{PdAu}_{5}{ }^{+}, \mathrm{PdAu}_{7}{ }^{+}, \mathrm{PdAu}_{9}{ }^{+}$, and $\mathrm{PdAu}_{11}{ }^{+}$. This additional channel is especially competitive for $\mathrm{PdAu}_{7}{ }^{+}$ and $\mathrm{PdAu}_{9}{ }^{+}$, in which cases Pd loss generates the stable closed-shell $\mathrm{Au}_{7}{ }^{+}$(6 electron) and $\mathrm{Au}_{9}{ }^{+}$(8 electron) clusters, respectively. For all other clusters, Au monomer evaporation is the only observed decay channel. It worth nothing that in Fig. 4, information on decay channels is only presented for $N \geqslant 6$. The small heat capacity of cluster sizes $N<6$ strongly suppresses metastable fragmentation.

The decay channels obtained from the experimental metastable fractions $M_{N}$ can be compared with dissociation energies calculated by DFT, which are also shown in Fig. 4(b) 
for $\mathrm{Au}_{N}{ }^{+}$and Fig. 4(d) for $\mathrm{PdAu}_{N-1}{ }^{+}$. The dissociation energy $\mathrm{D}_{N}$, of a cluster $A_{N}{ }^{+}$emitting a neutral fragment $A_{M}$, is defined as

$$
D_{N}=E\left(A_{N-M}^{+}\right)+E\left(A_{M}\right)-E\left(A_{N}^{+}\right) .
$$

In these calculations, the reactant $\left(A_{N}{ }^{+}\right)$and product $\left(A_{N-M}{ }^{+}\right)$cluster ions are the lowest-energy structures found in our BPGA search (Fig. 2) and have energies calculated by reoptimization with NWChem. The neutral atomic or diatomic fragments emitted $\left(A_{M}, M=1\right.$ or 2$)$ have also been energy minimized with NWChem. Only neutral monomers and dimers are considered in this analysis because the ionization energies of $\mathrm{Au}, \mathrm{Pd}, \mathrm{Au}_{2}$, and $\mathrm{PdAu}$ are all significantly higher than those of the larger gold and Pd-doped gold clusters. Thus, the lower-energy dissociation channels will be those where the positive charge resides on the larger cluster fragments, rather than on the evaporated monomers or dimers. The calculated $D_{N}$ 's for the monomer and dimer evaporation channels of $\mathrm{Au}_{N}{ }^{+}$and $\mathrm{PdAu}_{N-1}{ }^{+}$clusters are listed in the Supplemental Material [54].

Considering the monomer decay channel of $\mathrm{Au}_{N}{ }^{+}$clusters, a comparison of Figs. 4(a) and 4(b) shows excellent correlation between the measured metastable fractions $\left(M_{N}\right)$ and the calculated dissociation energies $\left(D_{N}\right)$. In the figure, the comparison is facilitated by plotting the opposite of $D_{N}$ since in the simpler case in which fragmentation proceeds mainly by one channel, a high (low) value of $D_{N}$ corresponds to a lower (higher) degree of metastable fragmentation. Turning to the dimer decay channel, we note that $D_{N}$ values for dimer loss are significantly higher than those for monomer loss for even values of $N$, which is consistent with the experimental finding that dimer evaporation is not observed for even $N$. For odd values of $N$, dissociation energies for monomer evaporation are high, reflecting the stability of these even-electron closed-shell species. As the $\mathrm{Au}_{2}$ dimer has an even number of electrons, dimer loss from a closed-shell cluster cation with an odd number of atoms generates a fragment which is also closed shell. Therefore, dissociation energies for dimer loss are mostly calculated to be lower than those for monomer evaporation, which is consistent with both decay channels being observed experimentally for odd $N$.

The calculated dissociation energies for monomer evaporation (loss of a single $\mathrm{Au}$ or $\mathrm{Pd}$ atom) from $\mathrm{PdAu}_{N-1}{ }^{+}$are shown in Fig. 4(d). The $D_{N}$ plot for Au loss shown in Fig. 4(d) does not exhibit the pronounced odd-even alternation observed experimentally for $M_{N}$ in Fig. 4(c), though $D_{N}$ values for Au evaporation are generally lower than for Pd evaporation, which is consistent with the experimental predominance of the $\mathrm{Au}$ evaporation channel. In contrast, a clear odd-even alternation in $D_{N}$ is calculated for the evaporation of a Pd atom up to $N=15$, with larger $D_{N}$ for odd $N$. For the Pd-doped clusters, a direct quantitative comparison between the experimental $M_{N}$ fractions and the calculated $D_{N}$ energies is not possible. Because the Pd-doped species have a complicated competition between the emission of different types of atoms, deriving an equation directly linking $M_{N}$ with $D_{N}$ is not possible [12]. However, a good prediction of the energetically most favorable fragmentation channels and for which cases Pd loss will compete with Au emission is achieved. As shown in Fig. 4(d),
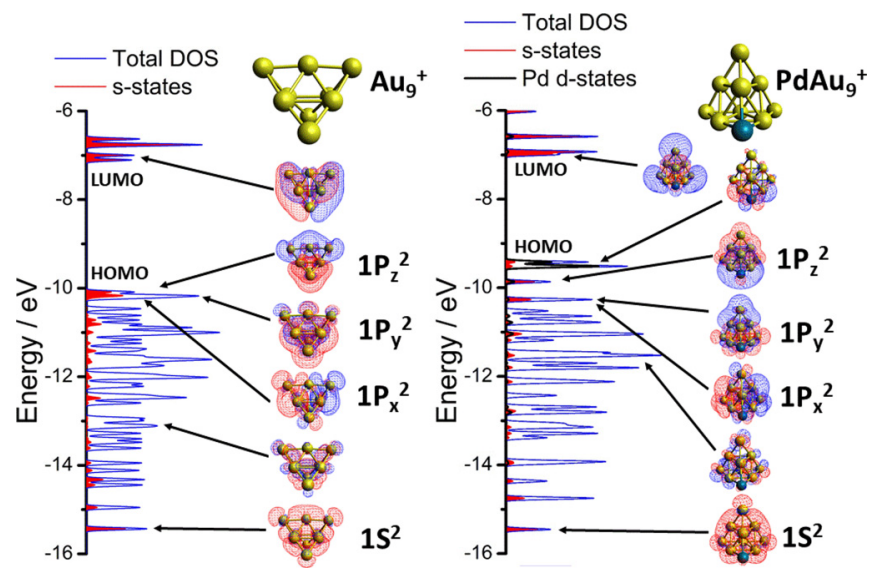

FIG. 5. Total density of states (blue line) of $\mathrm{Au}_{9}{ }^{+}$(left) and $\mathrm{PdAu}_{9}{ }^{+}$(right) clusters. The projection onto atomic $s$ states is shaded red, whereas the projection onto Pd $d$ states is shaded black. Molecular orbitals in which the electron density extends over the entire cluster are plotted and labeled based on their nodal character $\left(1 S\right.$ and $\left.1 P_{x, y, z}\right)$. In addition, a molecular orbital in which a higher electron density on the different atoms and a zero or close-to-zero electron density in between the atoms of the cluster is identified. The HOMO and LUMO levels are labeled.

$\operatorname{PdAu}_{N-1}{ }^{+}$clusters with even $N$ generally have similar $D_{N}$ energies for $\mathrm{Au}$ and Pd loss, in good agreement with the experimental $M_{N}$ fractions of Fig. 4(c). This is particularly noticeable for $\mathrm{PdAu}_{7}{ }^{+}(N=8)$ and $\mathrm{PdAu}_{9}{ }^{+}(N=10)$, in which Pd evaporation competes so that the very stable clusters $\mathrm{Au}_{7}{ }^{+}$and $\mathrm{Au}_{9}{ }^{+}$are formed. According to the fragmentation experiments, there is no clear evidence for the evaporation of $\mathrm{Au}_{2}$ or PdAu dimers from the Pd-doped clusters. As this may be due to experimental sensitivity problems, we have calculated the dissociation energies for evaporation of $\mathrm{Au}_{2}$ and $\mathrm{PdAu}$ from $\mathrm{PdAu}_{N}^{+}$(see Supplemental Material [54]). In most cases, calculations indicate that $D_{N}$ for dimer loss are higher than for monomer evaporation, with $\mathrm{Au}_{2}$ loss favored over $\mathrm{AuPd}$. It should be noted, however, that the calculated $D_{N}$ for the evaporation of $\mathrm{Au}_{2}$ from $\mathrm{PdAu}_{5}{ }^{+}$is only $1.81 \mathrm{eV}$. In fact, the calculated energies for the evaporation of $\mathrm{Au}$ and $\mathrm{Pd}$ atoms and the $\mathrm{Au}_{2}$ dimer from $\mathrm{PdAu}_{5}{ }^{+}$are very similar, lying in the range $1.81-1.99 \mathrm{eV}$, so all three fragmentation channels may be competitive.

\section{Electronic structure and magic numbers}

In the extended size range studied, four clusters are particularly interesting in view of their apparent higher stability: $\mathrm{Au}_{9}{ }^{+}, \mathrm{PdAu}_{9}{ }^{+}, \mathrm{Au}_{7}{ }^{+}$, and $\mathrm{PdAu}_{6}{ }^{+}$. To understand why these clusters exhibit higher stability than the other species, a detailed analysis of their electronic structures was performed.

The $5 d$ states in gold clusters are significantly hybridized with the valence $6 s$ states [7]. As a result, the use of simple electron-counting rules to interpret the size-dependent stability of gold clusters is more complicated than, for example, for $\mathrm{Na}$ clusters. This is illustrated in the left panel of Fig. 5, showing the total density of states (DOS) of $\mathrm{Au}_{9}{ }^{+}$. Below the highest occupied molecular orbital (HOMO), many states form a dense band, composed of hybridized $d$ and $s$ states, 
as a projection of the DOS reveals. At the top and bottom of this band, however, four states with high $s$ character are found, i.e., at around $-10 \mathrm{eV}$ (three states) and $-15.5 \mathrm{eV}$ (one state). Inspection of these MOs show wave functions delocalized over the entire cluster that, based on their nodal character [20], can be classified as the $1 S$ and $1 P_{x, y, z}$ eigenstates of the "particle in a spherical box" [64]. These MOs are plotted in the figure, together with a (randomly chosen) MO of mainly $d$ character, in which there is electron density localized on each atom of the cluster ("localized" here implies a MO with higher density on the different atoms and a zero or close-to-zero electron density in between the atoms). The doubly occupied $1 S$ and $1 P_{x, y, z}$ MOs (total of eight delocalized electrons) in the three-dimensional $\mathrm{Au}_{9}{ }^{+}$cluster form a closed electronic shell structure, providing enhanced stability for the cluster. It is worth mentioning that in a potential with perfect radial symmetry, the $1 P_{x}, 1 P_{y}$, and $1 P_{z}$ eigenstates are degenerate; however, because of the reduced symmetry of the actual cluster structure, as compared to a sphere, the degeneracy of the $1 P_{x, y, z}$ states is lifted (apart from in cubic and icosahedral point groups) so these three orbitals have slightly different energies in the cluster DOS [65].

The case of $\mathrm{PdAu}_{9}^{+}$, presented in the right panel of Fig. 5, is similar to that of $\mathrm{Au}_{9}{ }^{+}$. Between -10.5 and $-15 \mathrm{eV}$, many states of mainly $d$ character form a dense band, with states of larger $s$ contribution located below and above it. These states with large $s$ contribution are delocalized over the entire cluster and can, based on their nodal character, be classified as the $1 S$ and $1 P_{x, y, z}$ MOs. In addition, a projection of the total DOS onto $\operatorname{Pd} d$ states (black shading) reveals minor $\operatorname{Pd}(d)-\operatorname{Au}(d)$ hybridization and almost no $\operatorname{Pd}(d)-\operatorname{Au}(s)$ hybridization. This supports the picture that in $\mathrm{PdAu}_{9}{ }^{+}$, the $\mathrm{Pd}$ remains with all its $4 d^{10}$ electrons localized, whereas each Au atom delocalizes its $6 s$ electron, giving a total of eight delocalized electrons. Interestingly, above the $1 P_{z} \mathrm{MO}$, two occupied orbitals of mainly $\operatorname{Pd}(d)$ character are located, one of which is the highest occupied molecular orbital (HOMO) of the cluster. These states are poorly hybridized with $\operatorname{Au}(d)$ states due to a poor energy overlap, reducing the HOMO-lowest-unoccupied molecular orbital (LUMO) gap of $\mathrm{PdAu}_{9}{ }^{+}$as compared to $\mathrm{Au}_{9}{ }^{+}$.

A similar analysis of the cluster DOS was performed for $\mathrm{Au}_{7}{ }^{+}$and $\mathrm{PdAu}_{6}{ }^{+}$and is presented in Fig. 6. The lowest-energy isomer of $\mathrm{Au}_{7}{ }^{+}$(left panel of the figure) has a two-dimensional centered-hexagonal structure. This cluster has three doubly occupied MOs in which the wave function is delocalized over the entire cluster, resembling the lowest three eigenfunctions of a "particle in a circular box," based on their nodal character $\left(1 S\right.$ and $1 P_{x, y}$ ) [5]. In a 2D cluster, the $1 P_{z}$ orbital cannot be generated from a basis of $s$-type atomic orbitals so the electronic shell closure after filling $1 S$ and $1 P_{x, y}$ corresponds to six electrons $[6,66]$. In fact, the LUMO state of $\mathrm{Au}_{7}{ }^{+}$ resembles the 1D eigenstate of the particle in the circular box. Using the itinerant electron picture, each $\mathrm{Au}$ atom delocalizes its $6 s$ electron, providing $\mathrm{Au}_{7}{ }^{+}$with six itinerant electrons and enhanced stability. Similar arguments have been used to explain the high relative stability of specific flat boron clusters of different charge states $[67,68]$.

Finally, we discuss the interesting case of $\mathrm{PdAu}_{6}{ }^{+}$(right panel of Fig. 6). As for the previous clusters, the DOS is formed by a dense band of mainly $d$ character (the $d$ band),

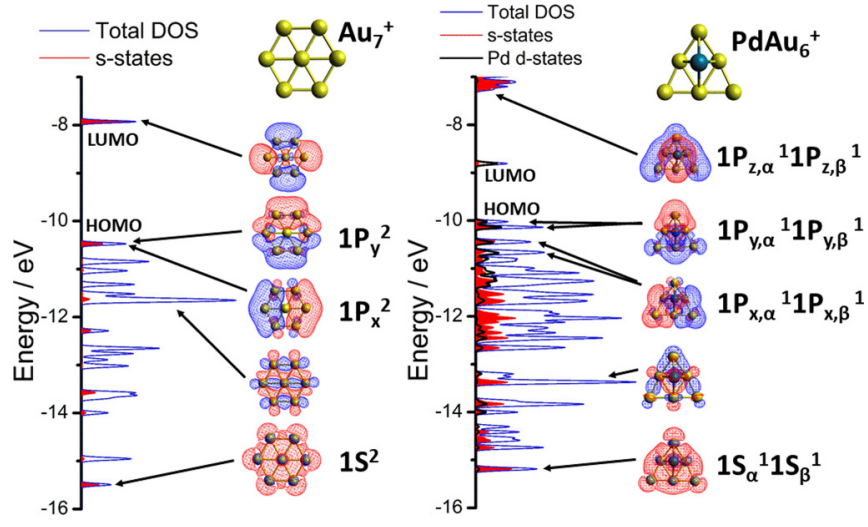

FIG. 6. Total density of states (blue line) of $\mathrm{Au}_{7}{ }^{+}$(left) and $\mathrm{PdAu}_{6}{ }^{+}$(right) clusters. A projection into atomic $s$ states is shaded red, whereas a projection onto Pd $d$ states is shaded black. Molecular orbitals in which the electron density extends over the entire cluster are plotted and labeled based on their nodal character $\left(1 S\right.$ and $\left.1 P_{x, y, z}\right)$. In addition, a molecular orbital in which a higher electron density on the different atoms and a zero or close-to-zero electron density in between the atoms of the cluster is identified. The HOMO and LUMO levels are labeled.

and six orbitals (counting $\alpha$ and $\beta$ orbitals for this odd-electron cluster) with a higher $s$ contribution. A plot of these states reveal MOs delocalized over the entire cluster, resembling the $1 S$ and $1 P_{x, y}$ eigenstates of the particle in a triangular box, based on their nodal character [6]. These states are doubly occupied, suggesting, in the itinerant electron model, that Pd delocalizes one of its $4 d^{10}$ electrons. Thus, $\mathrm{PdAu}_{6}{ }^{+}$has a total of six itinerant electrons, with a closed 2D electronic shell and enhanced stability. A similar conclusion was drawn from photoelectron spectroscopy measurements on small anionic $\operatorname{PdAu}_{N}{ }^{-}(N=1-4)$ clusters [21]. The very similar photoelectron spectra observed for anionic gold clusters and their Pd-doped equivalents suggested that in the doped clusters, one $4 d \mathrm{Pd}$ electron is promoted to a $5 s$ electronic level and participates in the bonding with Au. A projection of the DOS of $\mathrm{PdAu}_{6}{ }^{+}$onto $\mathrm{Pd} d$ states shows a very strong hybridization between $\operatorname{Pd}(d)$ and $\operatorname{Au}(s)-\operatorname{Au}(d)$ states. In particular, the $1 P_{x}$ and $1 P_{y}$ MOs of the cluster possess a significant percentage of $\mathrm{Pd}(d)$ character, in clear contrast to the case of $\mathrm{PdAu}_{9}{ }^{+}$. This supports the simple picture that Pd donates one of its $d$ electrons for delocalization and shell closing in the $\mathrm{PdAu}_{6}{ }^{+}$ cluster.

It should be noted, however, that the lowest-energy isomer of $\mathrm{PdAu}_{6}{ }^{+}$is not strictly two dimensional since the $\mathrm{Pd}$ atom occupies a site out of the plane defined by the six gold atoms. Nevertheless, delocalization takes place mostly in the plane of the Au atoms, and thus a quasi-2D picture remains: this can be understood by the well-known high stability of the neutral $\mathrm{Au}_{6} 2 \mathrm{D}$ triangular cluster [60]. $\mathrm{PdAu}_{6}{ }^{+}$can, therefore, reasonably be described as $\left(\mathrm{Pd}^{+}\right) \mathrm{Au}_{6}$. In $\mathrm{PdAu}_{6}{ }^{+}$, the $1 P_{z}$ orbital (which is now possible due to the overall $3 \mathrm{D}$ topology) is destabilized, opening a significant HOMO-LUMO gap in the system's DOS. This gap, however, is smaller than in $\mathrm{Au}_{9}{ }^{+}$ since, as shown in the figure, the LUMO state is a MO of mainly $\operatorname{Pd}(d)$ character, which is poorly hybridized with $\operatorname{Au}(d)$ due to a poor energy overlap with the $\mathrm{Au} d$ band. The enhanced 
stability of the odd-electron $\mathrm{PdAu}_{6}{ }^{+}$cluster, which is due to the even-electron nature of the $\mathrm{Au}_{6}$ triangle, is manifested in the local maximum in the second difference plot for $\mathrm{PdAu}_{6}{ }^{+}$ [Fig. 3(d)]. This also explains the reduced stability (relative to neighboring sizes) of the even-electron species $\mathrm{PdAu}_{5}{ }^{+}$and $\mathrm{PdAu}_{7}{ }^{+}$, as predicted by the DFT calculations [Fig. 3(d)] and confirmed by the experiments [Fig. 3(c)].

An interesting comparison that enables a better understanding of the enhanced stability of $\mathrm{PdAu}_{6}{ }^{+}$is the analysis of an isomer resembling the quasicircular structure of $\mathrm{Au}_{7}{ }^{+}$, comprising an Au hexagon with the Pd dopant at the center. A detailed analysis of this isomer, with a relative energy of +0.6 $\mathrm{eV}$ compared to the ground state, is presented in Appendix B. Remarkably, even though the Pd sits in the same plane as the Au framework, there is very weak hybridization between the $\operatorname{Pd}(d)$ and the $\operatorname{Au}(d)-\operatorname{Au}(s)$ states, similar to the situation in $\mathrm{PdAu}_{9}{ }^{+}$. As a consequence, of the MOs which are delocalized over the entire cluster, only the $1 S$ and $1 P_{x}$ MOs are doubly occupied, whereas the $1 P_{y}$ orbital is singly occupied. This implies a total of five itinerant electrons. Therefore, geometry has a determining role in the enhanced stability of $\mathrm{PdAu}_{6}{ }^{+}$, allowing good hybridization of the $d$ states of $\mathrm{Pd}$ with the $s$ and $d$ bands of Au only for the 3D isomer.

It is interesting to consider how much electron correlation and relativistic effects determine the stability patterns observed in these clusters. To address this, calculations were performed on the four clusters $\mathrm{Au}_{9}{ }^{+}, \mathrm{PdAu}_{9}{ }^{+}, \mathrm{Au}_{7}{ }^{+}$, and $\mathrm{PdAu}_{6}{ }^{+}$ using single-determinant Hartree-Fock (HF) theory, in which electron correlation and relativistic effects are excluded. At the HF level, MOs which are delocalized over the entire cluster can be found in the cluster DOS. Their energies are all above the $d$ band of the cluster. This is in sharp contrast with the DFT calculations discussed above, for which these delocalized orbitals are found within or even below (for the $1 S \mathrm{MO}$ ) the $d$ band. In the absence of relativistic effects, the $d$ band of $\mathrm{Au}$ is lower in energy and does not hybridize with the $s$ band. These results, shown in the Supplemental Material [54], can be expected to represent what would be observed, for instance, in Pd-doped Ag clusters, in which relativistic effects are much less important.

\section{CONCLUSIONS}

In this work, we have employed a combined experimental and theoretical approach to determine and rationalize the stability trends of small $\mathrm{Au}_{N}{ }^{+}$and $\mathrm{PdAu}_{N-1}{ }^{+}$clusters in the $N=5-20$ size range. The existence of "magic" clusters sizes at $\mathrm{Au}_{7}{ }^{+}, \mathrm{Au}_{9}{ }^{+}, \mathrm{PdAu}_{6}{ }^{+}$, and $\mathrm{PdAu}_{9}{ }^{+}$is observed in both intensity profiles from mass spectrometry and metastable photofragmentation fractions. The increased stability of these clusters is attributed to electronic shell closings in a 2D and 3D confining potential. The ground-state structures of $\mathrm{Au}_{7}{ }^{+}$and $\mathrm{PdAu}_{6}{ }^{+}$represent perturbations of the particle in a circular potential, which exhibit closed-shell electronic structures with six delocalized electrons, while $\mathrm{Au}_{9}{ }^{+}$and $\mathrm{PdAu}_{9}{ }^{+}$exhibit structures close to that of the particle in a spherical potential, with a corresponding closed eight-electron shell. These conclusions are supported by detailed analysis of the cluster density of states. Oscillations in the stability of nonmagic clusters are observed experimentally for all investigated clusters according to the possibility of closure of the $5 d$ orbital, with odd-numbered $\mathrm{Au}_{N}{ }^{+}$clusters, and even $\operatorname{PdAu}_{N}^{+}$clusters more stable. Excellent agreement is found with theory, suggesting that the calculated structures are indeed global minima and are representative of the clusters produced in the experiment. Moreover, photofragmentation fractions are compared with calculated dissociation energies, with qualitative agreement, showing that the balance between monomer and dimer evaporation is controlled by the ability to maintain closed $5 d$ shell daughter clusters. Furthermore, it is found that the evaporation of $\mathrm{Pd}$ atoms is suppressed in general, with exceptions for clusters in which this channel produces fragments of magic sizes.

In conclusion, we have demonstrated the significant effect of Pd doping on the stability and electron delocalization in small cationic Au clusters. These observations may be of relevance for the understanding of processes taking place in related systems, such as doped monolayer-protected clusters or larger bimetallic nanoparticles.

\section{ACKNOWLEDGMENTS}

This work was supported by the Research FoundationFlanders (FWO) and the KU Leuven Research Council (GOA/14/007). P.F. acknowledges the FWO for financial support through a postdoctoral grant. J.V. thanks the FWO for doctoral financial support. H.A.H. is grateful to the University of Kufa and the Ministry of Higher Education and Scientific Research (Iraq) for financial support through the award of a Ph.D. scholarship. Calculations were performed at the University of Birmingham's Blue-BEAR high-performance computer [69], and the UK's national HPC facility ARCHER, through membership of the HPC Materials Chemistry Consortium, funded by EPSRC (Grant No. EP/L000202), and EPSRC Critical Mass Grant (Grant No. EP/J010804) “TOUCAN: Towards an Understanding of Catalysis on Nanoalloys."

\section{APPENDIX A: DENSITY OF STATES OF PdAu ${ }_{7}{ }^{+}$AND $\mathrm{PdAu}_{8}{ }^{+}$CLUSTERS}

In the left panel of Fig. 7, the analysis of $\mathrm{PdAu}_{7}{ }^{+}$is presented. Four molecular orbitals in which the electron density is delocalized over the entire cluster can be identified in the cluster DOS. In clear contrast with $\mathrm{PdAu}_{9}{ }^{+}$(Fig. 5), the $1 P_{z}$ orbital is found to lie above the HOMO, and thus is empty. MOs $1 S$ and $1 P_{x, y}$ are doubly occupied, resulting in a total of six delocalized electrons. Since the cluster has a 3D geometry, the total number of six delocalized electrons does not correspond to a magic size. This is supported by the small HOMO-LUMO gap. The case of $\mathrm{PdAu}_{8}{ }^{+}$(right panel of Fig. 7) is similar to $\mathrm{PdAu}_{7}{ }^{+}$, with the difference that this cluster is an open-shell system and thus $\alpha$ - and $\beta$-spin orbitals must be considered independently. Four orbitals of delocalized character over the entire cluster are found in the cluster DOS, with only the $\beta-1 P_{z}$ orbital being empty. Therefore, $\mathrm{PdAu}_{8}{ }^{+}$has seven delocalized electrons 

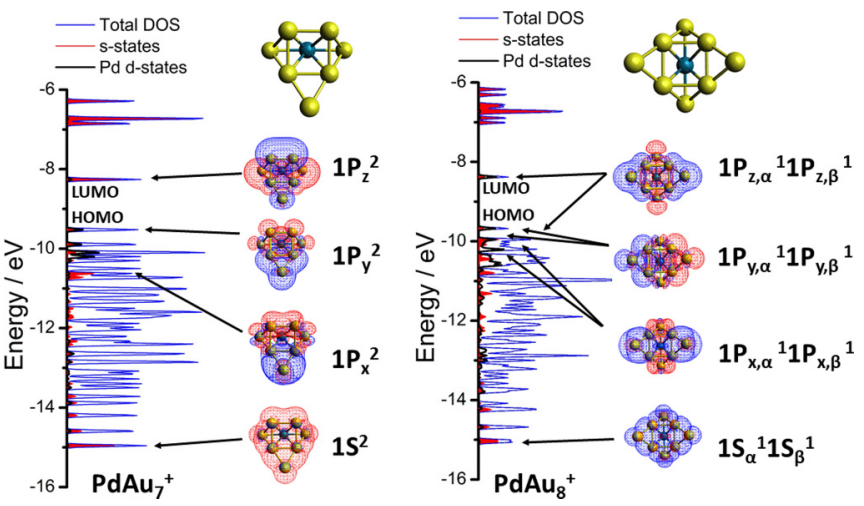

FIG. 7. Total density of states (blue line) of $\mathrm{PdAu}_{7}{ }^{+}$(left) and $\mathrm{PdAu}_{8}{ }^{+}$(right) clusters. A projection onto total $s$ states is shaded red, whereas a projection onto Pd $d$ states is shaded black. Molecular orbitals in which the electron density extends over the entire cluster are plotted and labeled based on their nodal character $\left(1 S\right.$ and $\left.1 P_{x, y, z}\right)$. The HOMO and LUMO levels are labeled.

\section{APPENDIX B: PLANAR ISOMER OF PdAu ${ }_{6}^{+}$}

This isomer, with a relative energy of $+0.6 \mathrm{eV}$ compared to the ground state, comprises an $\mathrm{Au}$ hexagon with the $\mathrm{Pd}$ dopant at the center. The total density of states is shown in Fig. 8, with a projection onto total $s$ and $\operatorname{Pd} d$ states. In this isomer, the hybridization of the $\mathrm{Pd} d$ states with $\mathrm{Au} d$ and $\mathrm{Au}$ $s$ states is poor, as in the case of $\mathrm{PdAu}_{9}{ }^{+}$presented in the main text. In fact, there are no MOs with high $\mathrm{Au} s$ character that simultaneously have large $\mathrm{Pd}$ contribution. This is reflected in the electronic structure of the isomer. The HOMO-LUMO gap of this cluster is very small since the $1 P_{y, \beta} \mathrm{MO}$ is empty. Thus, in the simplistic picture of itinerant electrons, $\mathrm{Pd}$ remains with all its $d$ electrons localized, resulting in a total of five electrons

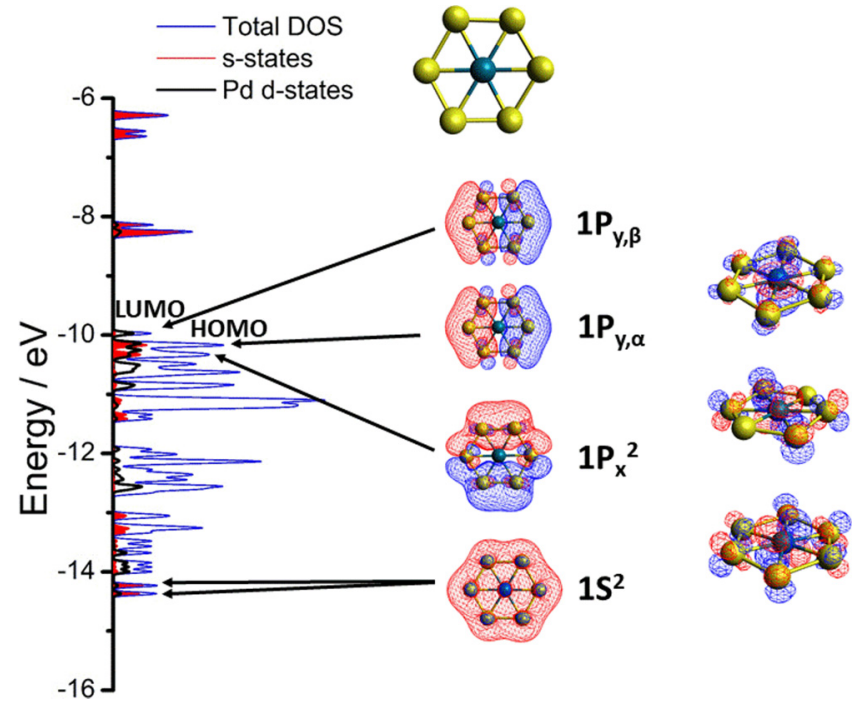

FIG. 8. Total density of states (blue line) of the planar hexagon isomer of $\mathrm{PdAu}_{6}{ }^{+}$, located $0.6 \mathrm{eV}$ above the ground state. A projection onto total $s$ states is shaded red, whereas a projection onto $\mathrm{Pd} d$ states is shaded black. Molecular orbitals in which the electron density extends over the entire cluster are plotted and labeled based on their nodal character $\left(1 S\right.$ and $\left.1 P_{x, y}\right)$. In addition, three molecular orbitals in which a higher electron density on the different atoms and a zero or closeto-zero electron density in between the atoms of the cluster is shown.

which are delocalized over the entire cluster. Therefore, geometry plays a determining role in the electronic structure of the cluster and, thus, in its stability. Interestingly, DFT calculations on the neutral cluster show that this hexagonal structure is the ground state for neutral $\mathrm{PdAu}_{6}$, instead of the triangular quasi-2D structure of the cation. By adding an extra electron, the system closes an electronic shell of delocalized valence electrons, providing enhanced stability for the planar structure.
[1] W. D. Knight, K. Clemenger, W. A. de Heer, W. A. Saunders, M. Y. Chou, and M. L. Cohen, Phys. Rev. Lett. 53, 510 (1984).

[2] H. Häkkinen, Chem. Soc. Rev. 37, 1847 (2008).

[3] A. C. Reber and S. N. Khanna, Acc. Chem. Res. 50, 255 (2017).

[4] W. A. de Heer, Rev. Mod. Phys. 65, 611 (1993).

[5] P. Atkins and R. Friedman, Molecular Quantum Mechanics (Oxford University Press, New York, 2011).

[6] E. Janssens, H. Tanaka, S. Neukermans, R. Silverans, and P. Lievens, New J. Phys. 5, 46 (2003).

[7] P. Pyykko, Chem. Rev. 88, 563 (1988).

[8] P. Gruene, D. M. Rayner, B. Redlich, A. F. G. van der Meer, J. T. Lyon, G. Meijer, and A. Fielicke, Science 321, 674 (2008).

[9] F. Furche, R. Ahlrichs, P. Weis, C. Jacob, S. Gilb, T. Bierweiler, and M. M. Kappes, J. Chem. Phys. 117, 6982 (2002).

[10] H. A. Hussein, I. Demiroglu, and R. L. Johnston, Eur. Phys. J. B 91, 34 (2018)

[11] S. Gilb, P. Weis, F. Furche, R. Ahlrichs, and M. M. Kappes, J. Chem. Phys. 116, 10 (2002).

[12] N. Veldeman, E. Janssens, K. Hansen, J. D. Haeck, R. E. Silverans, and P. Lievens, Faraday Discuss. 138, 147 (2008).
[13] M. Vogel, K. Hansen, A. Herlert, and L. Schweikhard, Phys. Rev. A 66, 033201 (2002).

[14] K. Hansen, A. Herlert, L. Schweikhard, and M. Vogel, Phys. Rev. A 73, 063202 (2006).

[15] H. Häkkinen, Adv. Phys. 1, 467 (2016).

[16] M. Haruta, T. Kobayashi, H. Sano, and N. Yamada, Chem. Lett. 16, 405 (1987).

[17] S. M. Lang, T. M. Bernhardt, R. N. Barnett, and U. Landman, Angew. Chem. Int. Ed. 49, 980 (2010).

[18] T. Fujitani, I. Nakamura, T. Akita, M. Okumura, and M. Haruta, Angew. Chem. Int. Ed. 48, 9515 (2009).

[19] S. Neukermans, E. Janssens, H. Tanaka, R. E. Silverans, and P. Lievens, Phys. Rev. Lett. 90, 033401 (2003).

[20] V. M. Medel, A. C. Reber, V. Chauhan, P. Sen, A. M. Köster, P. Calaminici, and S. N. Khanna, J. Am. Chem. Soc. 136, 8229 (2014).

[21] K. Koyasu, M. Mitsui, A. Nakajima, and K. Kaya, Chem. Phys. Lett. 358, 224 (2002).

[22] Z. J. Wu, S. H. Zhou, J. S. Shi, and S. Y. Zhang, Chem. Phys. Lett. 368, 153 (2003). 
[23] G. Zanti and D. Peeters, J. Phys. Chem. A 114, 10345 (2010).

[24] D. W. Yuan, Y. Wang, and Z. Zeng, J. Chem, Phys. 122, 114310 (2005).

[25] V. Kaydashev, P. Ferrari, C. Heard, E. Janssens, R. L. Johnston, and P. Lievens, Part. Part. Syst. Charact. 33, 364 (2016).

[26] V. E. Kaydashev, E. Janssens, and P. Lievens, J. Chem. Phys. 142, 034310 (2015).

[27] S. M. Lang, A. Frank, I. Fleischer, and T. M. Bernhardt, Eur. Phys. J. D 67, 19 (2013).

[28] L. Luo, Z. Duan, H. Li, J. Kim, G. Henkelman, and R. Crooks, J. Am. Chem. Soc. M. 139, 5538 (2017).

[29] S. Nishimura, Y. Yakita, M. Katayama, K. Higashimine, and K. Ebitani, Catal. Sci. Technol. 3, 351 (2013).

[30] G. J. Hutchings and C. J. Kiely, Acc. Chem. Res. 46, 1759 (2003).

[31] A. Bruma, F. R. Negreiros, S. Xie, T. Tsukuda, R. L. Johnston, A. Fortunelli, and Z. Y. Li, Nanoscale 5, 9620 (2013).

[32] S. Xie, H. Tsunoyama, W. Kurashige, Y. Negishi, and T. Tsukuda, ACS Catal. 2, 1519 (2012).

[33] Y. Negishi, W. Kurashige, Y. Niihori, T. Iwasa, and K. Nobusada, Phys. Chem. Chem. Phys. 12, 6219 (2010).

[34] K. A. Kacprzak, L. Lehtovaara, J. Akola, O. Lopez-Acevedo, and H. Häkkinen, Phys. Chem. Chem. Phys. 11, 7123 (2009).

[35] Y. Niihori, W. Kurashige, M. Matsuzaki, and Y. Negishi, Nanoscale 5, 508 (2013).

[36] P. Ferrari, J. Vanbuel, Y. L. T. Li, E. Janssens, and P. Lievens, in Gas Aggregation Synthesis of Nanoparticles, edited by Y. Huttel (Wiley-VCH, Berlin, 2017).

[37] E. Janssens, T. Van Hoof, N. Veldeman, S. Neukermans, M. Hou, and P. Lievens, Int. J. Mass Spectrom. 252, 38 (2006).

[38] S. Neukermans, X. Wang, N. Veldeman, E. Janssens, R. E. Silverman, and P. Lievens, Int. J. Mass Spectrom. 252, 145 (2006).

[39] https://bitbucket.org/JBADavis/bpga (unpublished).

[40] R. L. Johnston, Dalton Trans. 22, 4193 (2003).

[41] B. Bandow and B. Hartke, J. Phys. Chem. A 110, 5809 (2006).

[42] A. Shayeghi, D. Götz, J. B. A. Davis, R. Schäfer, and R. L. Johnston, Phys. Chem. Chem. Phys. 17, 2104 (2015).

[43] J. B. A. Davis, A. Shayeghi, S. L. Horswell, and R. L. Johnston, Nanoscale 7, 14032 (2015).

[44] G. Kresse and J. Hafner, Phys. Rev. B 47, 558 (1993).

[45] J. P. Perdew, K. Burke, and Y. Wang, Phys. Rev. B 54, 16533 (1996).

[46] G. Kresse and D. Joubert, Phys. Rev. B 59, 1758 (1999).

[47] M. Methfessel and A. T. Paxton, Phys. Rev. B 40, 3616 (1989).

[48] M. Valiev, E. Bylaska, N. Govind, K. Kowalski, T. Straatsma, and H. V. Dam, Comput. Phys. Commun. 1181, 1477 (2010).
[49] F. Weigend and R. Ahlrichs, Phys. Chem. Chem. Phys. 7, 3297 (2005)

[50] O. Vydrov and G. Scuseria, J. Chem. Phys. 125, 234109 (2006).

[51] M. Rohrdanz, K. Martins, and J. Herbert, J. Chem. Phys. 130, 054112 (2009).

[52] K. Hansen, Statistical Physics of Nanoparticles in the Gas Phase (Springer Science \& Business Media, Dordrecht, 2013).

[53] P. Ferrari, E. Janssens, P. Lievens, and K. Hansen, J. Chem. Phys. 143, 224313 (2015).

[54] See Supplemental Material at http://link.aps.org/supplemental/ 10.1103/PhysRevA.97.052508 for Hartree-Fock calculations for $\mathrm{PdAu}_{6}{ }^{+}, \mathrm{Au}_{7}{ }^{+}, \mathrm{PdAu}_{9}{ }^{+}$and $\mathrm{Au}_{9}{ }^{+}$; Determination of fragmentation channels; The calculated dissociation energies of the different fragmentation channels; and XYZ coordinates of minimum-energy structures.

[55] L. Ferrighi, B. Hammer, and G. K. H. Madsen, J. Am. Chem. Soc. 9, 10605 (2009).

[56] F. Remacle and E. S. Kryachko, J. Chem. Phys. 122, 044304 (2005).

[57] E. M. Fernández, J. M. Soler, I. L. Garzón, and L. C. Balbás, Phys. Rev. B 70, 165403 (2004).

[58] X. Wu, L. Senapati, and S. K. Nayak, J. Chem. Phys. 117, 4010 (2002).

[59] A. N. Gloess, H. Schneider, J. M. Weber, and M. M. Kappes, J. Chem. Phys. 128, 114312 (2008).

[60] H. A. Hussein, J. B. A. Davis, and R. L. Johnston, Phys. Chem. Chem. Phys. 18, 26133 (2016)

[61] C. J. Heard, A. Shayeghi, R. Schaefer, and R. L. Johnston, Z. Phys. Chem. 230, 955 (2016).

[62] Y. Chen, X. Kuang, X. Sheng, H. Wang, P. Shao, and M. Zhong, Z. Naturforsch. A 68, 651 (2013).

[63] E. M. Fernández and L. C. Balbás, J. Chem. Phys. 144, 224308 (2016).

[64] V. M. Medel, J. U. Reveles, S. N. Khanna, V. Chauhan, P. Sen, and A. W. Castleman, Proc. Natl. Acad Sci. USA 108, 10062 (2011).

[65] W. H. Blades, A. C. Reber, S. N. Khanna, L. López-Sosa, P. Calaminici, and A. M. Köster, J. Phys. Chem. A 121, 2990 (2017).

[66] E. Janssens, S. Neukermans, and P. Lievens, Curr. Opin. Solid State Mater. Sci. 8, 185 (2004).

[67] T. Ba Tai, R. W. A. Havenith, J. L. Teunissen, A. R. Dok, S. D. Hallaert, M. T. Nguyen, and A. Ceulemans, Inorg. Chem. 52, 10595 (2013).

[68] B. Kiran, G. G. Kumar, M. T. K. A. K. Nguyen, and P. Jena, Inorg. Chem. 48, 9965 (2009).

[69] http://www.birmingham.ac.uk/bear (unpublished). 